\title{
Isolation of Porcine Epidemic Diarrhea Virus during Outbreaks in South Korea, 2013-2014
}

\author{
Hee-Chun Chung, ${ }^{1}$ Van Giap Nguyen, ${ }^{1}$ \\ Hyoung-Joon Moon, Jee-Hoon Lee, \\ Seong-Jun Park, Ga-Eun Lee, Hye-Kwon Kim, \\ You-Shun Noh, Chan-Hee Lee, Dane Goede, \\ Bong Kyun Park
}

Author affiliations: Seoul National University, Seoul, South Korea (H.-C. Chung, J.-H. Lee, G.-E. Lee, Y.-S. Noh, C.-H. Lee, B.K. Park); Vietnam National University of Agriculture, Hanoi, Vietnam (V.G. Nguyen); Green Cross Veterinary Products, Yongin, South Korea (H.-J. Moon); National Forensic Service, Chilgok, South Korea (S.-J. Park); Institute for Basic Science, Daejeon, South Korea (H.-K. Kim); Korea Research Institute of Bioscience and Biotechnology, Daejeon, Republic of Korea (H.-K. Kim); University of Minnesota, St. Paul, Minnesota, USA (D. Goede)

\section{DOI: http://dx.doi.org/10.3201/eid2112.150437}

To the Editor: Porcine epidemic diarrhea (PED) is an acute infectious diarrhea caused by the PED virus (PEDV), which belongs to the order Nidovirales, family Coronaviridae, genus Alphacoronavirus (1). The virus is transmitted mainly through fecal-oral routes and infects all age groups of pigs; the most severe form of disease occurs in suckling piglets (1). PEDV was first reported in South Korea in 1992 (2), with the occurrence of an outbreak, and has since circulated with considerable genetic diversity $(1,3)$. During 2013, PED outbreaks reoccurred in South Korea; however, the emerging PEDVs in these outbreaks were not variants of previous Korean isolates or attenuated vaccine strains $(4,5)$. We report on a field isolate of a novel emerging PEDV and the isolate's genetic relationship with other PEDV strains.

During October 2013-June 2014, dead piglets and fecal swabs from 9 provinces of South Korea were sent to the Department of Veterinary Medicine Virology Laboratory at Seoul National University to confirm diagnoses of enteric viral diseases. All samples (30 intestine samples of dead piglets and 16 fecal swabs) were found to be PEDV positive. Attempts to isolate the field strains of PEDV on Vero cell lines followed a previously described protocol with modifications (6). An overnight monolayer of Vero cells (80\%-100\% confluence) was washed twice with $1 \times$ phosphate-buffered saline before homogenized samples $(0.02$ $\mu \mathrm{m}$ filtered) were inoculated with $10 \%$ suspension. After 30 min absorption at $37^{\circ} \mathrm{C}$ with $5 \% \mathrm{CO}_{2}$, maintenance medium (Dulbecco's Modified Eagle Medium supplemented

${ }^{1}$ These authors contributed equally to this article. with trypsin $[10 \mu \mathrm{g} / \mathrm{mL}])$, yeast extract $(0.04 \%)$, tryptose phosphate broth $(0.6 \%)$, and Antibiotic-Antimycotic $100^{\prime}$ (4 $\mu \mathrm{l} / \mathrm{mL}$; Gibco, Thermo Fisher Scientific, Grand Island, NY, USA) were added at a ratio of 1:10. The inoculated cells were cultured for $3-4$ days at $37^{\circ} \mathrm{C}$ in $5 \% \mathrm{CO}_{2}$ atmosphere and were blindly passaged 5 times. One field strain of PEDV (named BM1) was successfully adapted for growth on Vero cells. This virus was isolated from a 60sow farm (identified as BM farm) that had not vaccinated its animals against PEDV. Pigs of all ages from the farm showed clinical symptoms of diarrhea, and death occurred for $100 \%$ of suckling piglets and $10 \%$ of sows. Examination at necropsy revealed that the dead piglets from BM farm were covered with brown blotches of dried diarrheal feces and their stomachs were filled with undigested milk. Thin, translucent small intestines that contained yellow fluid were also observed (online Technical Appendix Figure 1, http:// wwwnc.cdc.gov/EID/article/21/12/15-0437-Techapp.pdf). The BM1 PEDV field isolate induced cytopathic effects of rounded shape (online Technical Appendix Figure 2, panel A) within 48 hours at passage 10. The presence of PEDV in the cell culture was confirmed by immunofluorescence assay (VDPro PEDV FA Reagent kit, MEDIAN Diagnostics, Gangwon-do, South Korea), which showed the specific fluorescence signal (online Technical Appendix Figure 2, panel B). In addition to evidence by microscopic observation, real-time reverse transcription PCR showed that the quantity of viral RNA increased incrementally as the number of passages increased: from 30,325 copies $/ \mu \mathrm{L}$ (cycle threshold 16.11) at passage 2 to 418,000 copies $/ \mu \mathrm{L}$ (cycle threshold 13.77) at passage 10. Infective titers of the BM1 isolate increased from $10^{4.7} 50 \%$ tissue culture infectious doses $/ \mathrm{mL}$ at passage 2 to $10^{7.9} 50 \%$ tissue culture infectious doses/mL at passage 10 (online Technical Appendix; online Technical Appendix Table 2).

The complete S gene of BM1 (GenBank accession no. KP861982) was sequenced for genetic characterization; the gene was 4,161-nt long and encoded 1,386 aa. The spike protein of the BM1 isolate showed substitutions at neutralizing SS6 epitope from LQDGQVKI (7) to SQSGQVKI but identity at the SS2 (7) and $2 \mathrm{C} 10(8)$ neutralizing epitopes. The genetic relationship of the BM1 isolate with other PEDVs in the world was inferred from a codon-based alignment of 409 sequences of the complete $\mathrm{S}$ gene (online Technical Appendix Table 3). The maximum-likelihood phylogenetic tree was constructed by using the FastTree program (9), with the general time reversible nucleotide substitution model. The phylogeny constructed on the basis of the complete $\mathrm{S}$ gene (Figure) showed that the BM1 isolate belongs to subgroup 2a, genogroup 2 of PEDV. This isolate clustered closely with emergent PEDV strains in the United States (online Technical Appendix Figure 3), showing $99.2 \%-99.7 \%$ identity with PEDVs of North American 


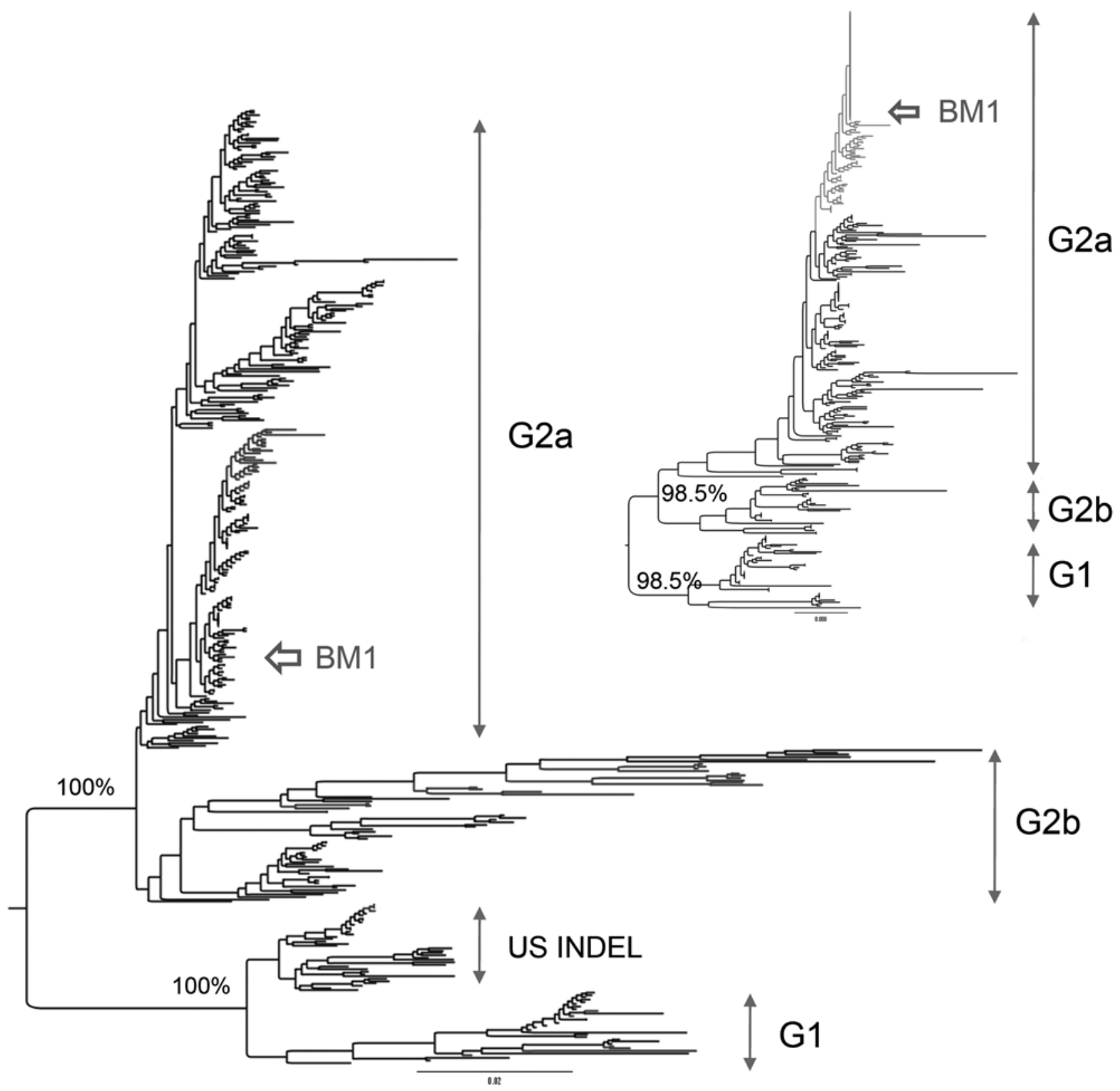

Figure. Maximum-likelihood phylogenetic tree of porcine epidemic diarrhea virus from piglet, South Korea, 2013-2014, constructed on the basis of codon alignment of complete $\mathrm{S}$ genes. Inset shows a phylogenetic tree inferred from the complete $\mathrm{N}$ genes. Genogroups are shown to the right of each tree. US INDEL is a prototype strain of porcine epidemic diarrhea virus that has insertions and deletions (INDELs) in the spike gene. Scale bars indicate nucleotide substitutions per site. A color version of this figure is available online (http://wwwnc.cdc.gov/eid/article/21/2/15-0437-F.htm).

strains (10). This observation was repeated by the phylogenetic inference of the complete $\mathrm{N}$ gene (Figure; online Technical Appendix Table 4 and Figure 4). The branching pattern (Figure) clearly showed that BM1 is genetically less related (92.9-93.4\% identity) to the live vaccine strains that are derived from genogroup 1 and used currently to prevent PEDV infections in South Korea.
In summary, we isolated the BM1 strain (GenBank accession no. KP861982) in South Korea from a sample from a suckling pig with severe diarrhea; the pig came from a farm that had not vaccinated its pigs against PEDV. The strain was adapted and grew to high titers on Vero cells. The isolate belongs to genogroup 2 and genetically clustered with emerging PEDVs of North American 
strains but was loosely related to genogroup 1, the basis of the vaccine used for inoculation against Korean PEDV strains. This isolate may need further evaluation as a candidate for a vaccine to prevent reemerging PEDVs in South Korea.

\section{Acknowledgments}

This study was supported by the BioGreen 21 Program, Rural Development Administration (grant no. PJ011184), and by the Bio-industry Technology Development Program (grant no. 114055031SB010), Ministry of Agriculture, Food and Rural Affairs, South Korea.

\section{References}

1. Song D, Park B. Porcine epidemic diarrhoea virus: a comprehensive review of molecular epidemiology, diagnosis, and vaccines. Virus Genes. 2012;44:167-75. http://dx.doi.org/10.1007/ s11262-012-0713-1

2. Kweon CH, Kwon BJ, Jung TS, Kee YJ, Hur DH, Hwang EK, et al. Isolation of porcine epidemic diarrhea virus (PEDV) in Korea. Korean J Vet Res. 1993;33:249-54.

3. Choi JC, Lee K, Pi J, Park S, Song C, Choi I, et al. Comparative genome analysis and molecular epidemiology of the reemerging porcine epidemic diarrhea virus strains isolated in Korea. Infect Genet Evol. 2014;26:348-51. http://dx.doi.org/10.1016/ j.meegid.2014.06.005

4. Park S, Kim S, Song D, Park B. Novel porcine epidemic diarrhea virus variant with large genomic deletion, South Korea. Emerg Infect Dis. 2014;20:2089-92. http://dx.doi.org/10.3201/ eid2012.131642

5. Lee S, Lee C. Outbreak-related porcine epidemic diarrhea virus strains similar to US strains, South Korea, 2013. Emerg Infect Dis. 2014;20:1223-6. http://dx.doi.org/10.3201/eid2007.140294

6. Chen Q, Li G, Stasko J, Thomas JT, Stensland WR, Pillatzki AE, et al. Isolation and characterization of porcine epidemic diarrhea viruses associated with the 2013 disease outbreak among swine in the United States. J Clin Microbiol. 2014;52:234-43. http://dx.doi.org/10.1128/JCM.02820-13

7. Sun D, Feng L, Shi H, Chen J, Cui X, Chen H, et al. Identification of two novel $\mathrm{B}$ cell epitopes on porcine epidemic diarrhea virus spike protein. Vet Microbiol. 2008;131:73-81. http://dx.doi.org/10.1016/j.vetmic.2008.02.022

8. Cruz DJ, Kim CJ, Shin HJ. Phage-displayed peptides having antigenic similarities with porcine epidemic diarrhea virus (PEDV) neutralizing epitopes. Virology. 2006;254:28-34. http://dx.doi.org/ 10.1016/j.virol.2006.04.027

9. Price MN, Dehal PS, Arkin AP. FastTree 2-approximately maximum-likelihood trees for large alignments. PLoS ONE. 2010;5:e9490. http://dx.doi.org/10.1371/journal.pone.0009490

10. Vlasova AN, Marthaler D, Wang Q, Culhane MR, Rossow KD, Rovira A, et al. Distinct characteristics and complex evolution of PEDV strains, North America. Emerg Infect Dis. 2014;20:1620-8.

Address for correspondence: Bong Kyun Park, Department of Veterinary Medicine Virology Laboratory, College of Veterinary Medicine and Research Institute for Veterinary Science, Seoul National University, Seoul 151-742, South Korea; email address: parkx026@snu.ac.kr

\section{Tickborne Lymphadenopathy Complicated by Acute Myopericarditis, Spain}

\author{
José Tiago Silva, Francisco López-Medrano, \\ Mario Fernández-Ruiz, Elena Resino Foz, \\ Aránzazu Portillo, José A. Oteo, \\ José Maria Aguado
}

Author affiliations: Instituto de Investigación Hospital "12 de Octubre" (i+12), Madrid, Spain (J.T. Silva, F. López-Medrano, M. Fernández-Ruiz, E.R. Foz, J.M. Aguado); Centro de Investigación Biomédica de La Rioja, Logroño, Spain (A. Portillo, J.A. Oteo)

DOI: http://dx.doi.org/10.3201/eid2112.150672

To the Editor: Dermacentor-borne necrosis erythema lymphadenopathy/tickborne lymphadenopathy (DEBONEL/TIBOLA) is an apparently benign, self-limiting rickettsial disease transmitted by Dermacentor ticks $(1,2)$. Rickettsia slovaca was the first etiologic agent isolated, but other species, such as $R$. raoultii and Candidatus R. rioja, also might be involved (3-6). If the scalp is affected, a larger number of agents (including Francisella tularensis, Bartonella henselae, $R$. massiliae, $R$. sibirica mongolitimonae, and Borrelia burgdorferi) should be considered within the differential diagnosis of a similar syndrome recently named scalp eschar associated with neck lymphadenopathy after a tick bite (SENLAT) (7). Nevertheless, in Spain, only R. slovaca, Candidatus $R$. rioja, and F. tularensis are known to cause DEBONEL/TIBOLA/SENLAT $(4,6)$. This entity is considered an emerging rickettsiosis in Europe; cases have been reported from Italy, France, Hungary, Germany, and Portugal (8).

We recently saw a patient in whom acute myopericarditis developed after he was bitten by a large tick on the scalp and showed clinical signs of DEBONEL/TIBOLA/ SENLAT, most likely attributable to $R$. slovaca or Candidatus R. rioja infection. The patient, a previously healthy 28-year-old man, went on a day-long hiking trip to the northern mountains of Madrid (central Spain; mean altitude $1,300 \mathrm{~m}$ ) on November 2, 2014. Three days later, he noticed a mild ache on the occipital area of his scalp and found an attached tick that he removed with his fingers. A week later, he sought care from an infectious disease specialist because of itchy discomfort at the area of the tick bite.

Examination revealed an erythematous and elevated punctiform lesion with mild fluctuation in the occipital region accompanied by tender, small lymph node enlargement of both occipital lymphatic chains (Figure). No widespread rash was present. DEBONEL/TIBOLA/SENLAT was diagnosed, and doxycycline (100 mg every 12 hours) was initiated. IgG titer against spotted fever group Rickettsia 